\title{
LAS CURVAS DE OFERTA Y DEMANDA DE FLEEMING JENKIN *
}

CARLOS RODRIGUEZ BRAUN

Universidad Complutense

\section{RESUMEN}

Fleeming Jenkin (1833-1885) fue el primer británico que dibujó curvas de oferta y demanda, las analizó como funciones matemáticas y las empleó para abordar problemas económicos. Célebre ingeniero, efectuó notables aportaciones a la economía, que fueron en su tiempo poco destacadas. Este artículo revisa el pensamiento de Jenkin, poniendo el énfasis en su análisis de las tres leyes de la oferta y la demanda, y su aplicación al mercado de trabajo y a los impuestos, que dio lugar al descubrimiento independiente de la noción de excedente del consumidor y del productor. El articulo sostiene que una razón por la que Jenkin no obtuvo el reconocimiento que merecía fue el trato hostil que le dispensaron Jevons y Marshall, que devaluaron sus contribuciones e insistieron en que se le habian anticipado. El articulo concluye que esta reivindicación carece de fundamento.

\section{ABSTRACT}

Fleeming Jenkin (1833-1885) was the first person in Britain to draw supply and demand curves, discussing them as mathematical functions and employing them to study economic problems. A celebrated engineer, he made remarkable contributions to eco-

* Este trabajo deriva de la primera parte del proyecto de investigación que presenté en las oposiciones a cátedra en la Universidad Complutense en abril de 1994. Agradezco los comentarios de los miembros del tribunal, y también los que me formularon con anterioridad Fernando Méndez Ibisate y los asistentes al seminario de la Universidad Complutense sobre los cien años de los Principios de Marshall, y con posterioridad Maria Blanco González, Luis Julián Álvarez Gonzälez y los integrantes del seminario de Historia Económica de la Universidad Carlos III. Agradezco en particular las criticas formuladas por dos evaluadores anónimos. 
nomics, that attracted little attention in his time. This article goes through Jenkin's thought, emphasizing his analysis of the three laws of supply and demand, and its application to the labour market and the taxes, that produced the independent discovery of the notion of consumer and producer surplus. The article holds that a reason why Jenkin lacked the acknowledgement he deserved lay in the hostile attitude towards him by Jevons and Marshall, who undervalued his contributions and insisted that they had anticipated Jenkin. The article concludes that this claim is unfounded.

\section{INTRODUCCIÓN}

La presente investigación discute la recepción de las teorias económicas de Fleeming ${ }^{1}$ Jenkin (1833-1885), el primer británico que publicó curvas de oferta y demanda, tratándolas explícitamente como funciones, y defiende la tesis de que sus méritos analíticos no fueron reconocidos adecuadamente en su momento por la injustificada hostilidad de Jevons y Marshall, que alegaron precedencia sobre Jenkin.

Aunque la importancia de Jenkin ha sido ponderada y admitida en la historiografía del pensamiento económico en el siglo xx, la recepción de sus ideas en su tiempo no ha recibido mucha atención. Este articulo examina críticamente las fuentes originales y la bibliografia secundaria para argumentar que la precedencia de Jevons y Marshall sobre Jenkin, que les llevó a devaluar la significación del análisis gráfico de éste sobre la oferta y la demanda, se basa sólo en pruebas testimoniales y no sustantivas. Mientras que la relación Jevons/Jenkin ha recibido algún tratamiento historiográfico, aunque sin un desenlace concluyente, la relación Marshall/Jenkin no ha sido prácticamente tratada, cuando en realidad es mucho más importante que la anterior a los efectos de la recepción de las ideas de Jenkin entre los economistas, puesto que el peso profesional de Marshall fue considerablemente mayor al de Jevons. Tras la introducción, que incluye una breve reseña biográfica de Jenkin, el articulo tiene dos partes. En la primera, titulada «Jenkin, Economista», se resumen las aportaciones de Jenkin, como marco de referencia para la discusión posterior. En la segunda parte, titulada «Jenkin y los Economistas», se entra en el debate y se estudian las reacciones que ante la obra de Jenkin adoptó la profesión económica.

Jenkin es uno de esos economistas importados de otras disciplinas, cuyas contribuciones al análisis económico no constituyen condición necesaria para que accedan al podio de los pensadores ilustres. Demostración de ello son las

\footnotetext{
1 El nombre debe pronunciarse «fleming» y no «fliming»; Stevenson (1887), p. 21
} 
24 líneas que recibe en la Enciclopedia Británica, en las que la palabra economia no aparece ni una sola vez. Según la cultura general, entonces, Jenkin fue un ingeniero especializado en electricidad. Henry Charles Fleeming Jenkin nació cerca de Dungeness, Kent, Inglaterra, el 25 de marzo de $1833^{2}$. En 1850 se graduó como ingeniero en la Universidad de Génova. Trabajó en empresas de ingeniería ferroviaria, eléctrica y de comunicaciones. Jenkin empezó a publicar trabajos técnicos animado por sir William Thomson, más conocido como lord Kelvin. Fue nombrado catedrático de ingeniería en el University College de Londres en 1866 y en la Universidad de Edimburgo en 1868. Fue entonces cuando comenzó a escribir sobre economía. En 1873 publicó su libro más famoso, Magnetismo y electricidad, traducido a varios idiomas. Murió el 12 de junio de 1885, en Edimburgo.

\section{JENKIN, ECONOMISTA}

Fleeming Jenkin merece figurar en la historia del pensamiento económico por tres artículos: «Legitimidad de los sindicatos» (1868), «La representación gráfica de las leyes de oferta y demanda y su aplicación al trabajo» (1870) y «Los principios que regulan la incidencia de los impuestos» (1871).

El primer trabajo aborda la cuestión sindical. Jenkin comenta un informe de la Comisión Real nombrada en 1867 para estudiar el problema laboral, después de unos graves incidentes que habían tenido lugar en Sheffield. Dicha circunstancia provocó la publicación de varios estudios sobre el asunto. Uno de ellos, el de William Thomas Thornton, dio lugar a un conocido episodio en la historia del pensamiento económico: la «retractación» de John Stuart Mill sobre la teoría del fondo de salarios.

Jenkin se plantea la pregunta de si pueden los sindicatos aumentar los salarios, y afronta dos respuestas negativas que se daban entonces, fundadas en el fondo de salarios y en la oferta y la demanda de trabajo. Descarta al fondo de salarios por una debilidad central: el fondo no es fijo. Analiza entonces algebraicamente la oferta y la demanda. Distingue entre cantidades ofertadas y demandadas y disposiciones subjetivas a demandar y ofrecer, para demostrar que el precio puede cambiar sin que lo hagan las cantidades: este resultado requiere «que cuando surge una aversión a ofertar el artículo al precio antiguo, surja también una inclinación por parte de los compradores a comprar a un precio

2 Para una extensa y notable biografia, véase Stevenson (1887). Fue muy afortunado Jenkin al contar entre sus alumnos y admiradores al autor de La isla del tesoro. 
mayor» ${ }^{3}$. En términos modernos, un desplazamiento hacia la izquierda de la curva de oferta y hacia la derecha de la curva de la demanda. Los factores subjetivos impiden la relación automática entre precio y cantidad, entre salarios y empleo. Las consecuencias de la igualación de oferta y demanda no pueden preverse, porque los motivos detrás de ellas son múltiples e impredecibles: el precio depende de la negociación y ello abre la puerta a los sindicatos ${ }^{4}$.

El segundo artículo de Jenkin analiza a la oferta y la demanda gráfica y no algebraicamente, y se resume en tres leyes que guardan interesantes paralelismos con los periodos detallados por Marshall e implícitamente presentes en la economía clásica.

Jenkin sigue la convención matemática usual — que cambiaría a partir de Marshall- de asignar a la variable dependiente el eje de ordenadas y a la variable independiente el eje de abcisas. Aunque algunos historiadores han sugerido que Jenkin maneja las curvas al estilo de Marshall 5, es más correcto afirmar que su enfoque en este punto es del tipo de Walras. Las curvas de Jenkin, en efecto, indican cantidades que los agentes están dispuestos a vender y comprar a cada precio, y el equilibrio se alcanza mediante variaciones en el precio. Ese equilibrio se produce cuando las curvas se cruzan; teóricamente, porque en la realidad las curvas son desconocidas y se van descubriendo a través de la competencia ${ }^{6}$. Jenkin no desarrolla las condiciones de estabilidad del equilibrio, pero los casos que discute no son de inestabilidad: la primera derivada de sus funciones de demanda no es positiva y la de sus funciones de oferta no es negativa. La primera ley de la oferta y la demanda es que el precio de mercado viene dado por el punto de cruce de las dos curvas. Es sabido que hubo geometría de la oferta y la demanda antes que Marshall: el primero que dibujó esas curvas fue Cournot en 1838; pero el tratamiento gráfico de Jenkin se pare-

3 Jenkin (1887), pp. 13,17n, 18, 18n, 19n.

4 Ibid., pp. 18, 22. El derecho a la libre sindicación es para Jenkin claro -nótese que en su tiempo dicha opinión no estaba extendida todavia, ni mucho menos- y es equivalente al derecho de los empresarios para hacer lo propio. La asimetría entre ambos derechos ya había sido se. ñalada por Adam Smith (1776), pp. 110-12 y 207-8. Los economistas clásicos fueron en general simpatizantes de los sindicatos, a pesar de los rasgos violentos de las asociaciones de trabajadores. Esta actitud de respaldo a los sindicatos se mantuvo después, con Alfred Marshall. Cf. O'Brien (1989), pp. 394.6, y Petridis (1973), p. 167. Véase también Groenewegen (1994). Una critica a esta actitud de los economistas, en Hutt (1936).

5 Brownlie y Lloyd Prichard (1963), pp. 208 y ss.

6 Hay más elementos walrasianos: «Si cada persona declarase por escrito de antemano exactamente cuánto venderá o comprará a cada precio, entonces el precio de mercado se determina. ría de inmediato y las transacciones se acordarian alli y entonces. En la práctica, el precio al que se venderá cada cuartal será una mera aproximación tentativa al precio teóricon; Jenkin (1887), p. 79 . 
cía más al que después publicaría Marshall que el de Cournot. Asimismo, para mayor mérito de Jenkin, no conoció la obra de sus predecesores ${ }^{7}$.

Lo expuesto hasta aquí plantea la cuestión de la subjetividad del valor y por lo tanto de la utilidad. En el artículo sobre los sindicatos Jenkin apunta que oferta y demanda sólo son inteligibles en términos de cantidades, pero que existe también una dimensión subjetiva - particularmente en el caso de la deman. da ${ }^{8}$ - que se debe distinguir de la otra, porque es impredecible. Pero añade: «Estas dos ecuaciones, la primera entre dos cantidades y la segunda entre dos valores, son verdaderas ambas, y una puede ser deducida de la otra». Jenkin no explora esta deducción porque no cree que pueda decirse nada de lo subjetivo ${ }^{9}$.

Algunos autores han destacado que la relación inversa entre cantidad y precio de la ecuación $D=f(A+1 / x)$, que Jenkin presentó en su artículo sobre los sindicatos, implica la utilidad marginal decreciente, pero Jenkin es muy parco al respecto. La expresión «utilidad» es empleada en una oportunidad y con cautela: «La demanda en la mente de los compradores se corresponde con la utilidad que a su juicio tiene el artículo; y las causas que confluyen para formar esa opinión son demasiado numerosas como para admitir una clasificación» ${ }^{10}$. Esta ausencia en última instancia de la utilidad en la explicación del valor deja a Jenkin atrás de sus contemporáneos Jevons, Menger y Walras, y lo excluye de la escuela de la utilidad marginal ${ }^{11}$.

La dimensión subjetiva del valor permite a Jenkin acotar la validez de la primera ley de la oferta y la demanda: como las mentes de las personas no se quedan quietas, las curvas podrán cambiar dentro de dos límites, la «oferta total» o cantidad total disponible y afondo de compra» o cantidad de dinero que los agentes disponen para comprar ${ }^{12}$. El paso siguiente es suponer constante a una de dichas magnitudes y aumentar la otra. Jenkin dibuja nuevas curvas para representar a la segunda ley: si la oferta total aumenta, los precios bajan, y si el fondo de compra aumenta, los precios suben.

7 Humphrey (1992), p. 21. Agradezco al profesor Fernando Méndez Ibisate por haber llamado mi atención hacia este articulo.

8 Jenkin (1887), p. 15. Según Jenkin, oferta es una expresión que se emplea "casi siempre» para referirse a una cantidad presentada a la venta. No obstante, también se la puede considerar como disposición subjetiva a vender; cf. Brownlie y Prichard (1963), pp. 208-9.

9 Jenkin (1887), p. 16. En varias ocasiones Jenkin subraya la importancia de la dimensión subjetiva del valor para prevenir ante las conclusiones demasiado rigidas y apresuradas del análisis de la oferta y la demanda. Ibid., pp. $87,88,93,95$. En su curioso manuscrito de 1884 , publicado póstumamente, "¿Es el beneficio de un hombre la pérdida de otro?», vuelve a destacar la subjetividad del valor: «es sólo el pensamiento lo que causa el valor de las cosas», p. 141.

10 Brownlie y Prichard (1963), p. 209; Jenkin (1887), p. 98; Uemiya (1981), p. 177.

11 Howey (1989), pp. 22, 86.

12 Jenkin (1887), p. 78. 
Jenkin sostiene que esto no es una ley en sentido estricto sino que expresa «un grado de probabilidad, que variará inmensamente según los casos y los bienes» ${ }^{13}$. En la realidad los mercados no funcionan como sus primeros gráficos los pintan, en particular el mercado de trabajo. Presenta entonces varios gráficos de mercados no competitivos, siguiendo a W. T. Thornton ${ }^{14}$. Pinta por ejemplo una oferta infinitamente inelástica y una demanda superpuesta en un tramo, lo que da como resultado un segmento de precios de equilibrio. Jenkin recuerda que tanto la primera ley como la segunda suponen que los individuos perciben la brecha entre las curvas a cada precio y que dichas leyes no pueden afectar a los casos en los que «compradores y vendedores no pueden estimar la relación entre la demanda a un precio y la oferta a un precio». Jenkin apunta que uno de esos casos es el del monopolio bilateral, por el que Edgeworth criticará a Jevons en 188115.

Jenkin presenta a continuación su tercera ley, integrada como las anteriores en la tradición clásica: a largo plazo el coste de producción determina el precio y la demanda la cantidad ${ }^{16}$. Una diferencia esencial entre el largo plazo y el corto es que las curvas de oferta y demanda medias a largo plazo pueden ser dibujadas con base empírica, a partir de las estadísticas históricas de ventas y de costes. No obstante, esas estadísticas permitirán trazar curvas sólo aproximadas, y no existe una ley para todos los bienes. En general, afirma Jenkin, las curvas de demanda tenderán a ser horizontales (o sea, con ejes al estilo moderno, inelásticas) y las de oferta tenderán a ser verticales (o sea, elásticas), pero puede haber casos donde la curvas tengan una elasticidad ampliamente diferente.

Jenkin traslada el análisis al mercado de trabajo. El salario es el precio en ese mercado, sujeto por tanto a las leyes de la oferta y la demanda, pero Jenkin añade que de ahi no se deduce que el salario dependa de una «ley natural» ${ }^{17}$.

13 Ibid., p. 81.

14 William Thomas Thornton publicó en 1869 On labour, donde critica la idea del fondo de salarios fijo e insiste en que los salarios son determinados sólo por la negociación. John Stuart Mill, compañero de trabajo de Thornton en la Compañia Inglesa de las Indias Orientales, se uretractó» dramáticamente en 1869 , en un artículo sobre On labour titulado «Thornton on labour and its claims». Parece que no fueron las criticas de Thornton sobre las leyes de la oferta y la demanda las que impresionaron a Mill sino las posibilidades que se abrian ante los sindicatos con la ausencia de un fondo de salarios fijo. Es posible que haya sido Mill quien influyó sobre Jenkin en este artículo publicado un año más tarde. Jenkin, no obstante, insiste en que él habia demos. trado la falacia del fondo de salarios en su trabajo de 1868 , «y la misma argumentación, empleada posteriormente por el Sr. Thornton, ha sido reconocida como acertada por el Sr. John Stuart Mill». Cf. Schumpeter (1954), pp. 669-70; Forget (1991); Gordon (1973); Negishi (1986); Ekelund y Thommesen (1989); Jenkin (1887), p. 94; Mill (1967), p. 635; Schwartz (1972), pp. 90-101, 274.

15 Jenkin (1887), p. 86; Creedy (1992), p. 172.

16 Jenkin (1887), p. 89.

17 Ibid., pp. 93-94. 
Como no hay curvas de demanda fijas, puesto que dependen de «estados mentales», la solución adecuada es dejar a los sindicatos libres y confiar en su propio interés, porque Jenkin reconoce que el poder negociador para subir los salarios puede conspirar contra el aumento del empleo.

Para conocer el nivel salarial hay que recurrir, como en los demás bienes, a la tercera ley. Pero el coste de producción del trabajo, su manutención, no es fijo. Si un trabajador cuenta con reservas - proporcionadas por su sindicatopara negociar con su empleador, pedirá más dinero: su coste de producción habrá aumentado. Si el trabajador aspira a un mayor nivel de vida ello automáticamente significa un mayor coste de producción: «El coste de producción del trabajo determina los salarios, pero ese coste a su vez es determinado por las expectativas de las personas sobre su nivel de vida» ${ }^{18}$. Los trabajadores determinan sus salarios: el coste de producción determina el precio. Ahora bien, el vendedor fija el precio pero el comprador la cantidad. Así, es el capital el que determina cuántos trabajadores se emplearán a cada tipo de salario. De ahi la importancia de la sabiduria sindical.

El tercer artículo de Jenkin, de 1871, estudia la incidencia de los impuestos con curvas de oferta y demanda ${ }^{19}$. Jenkin menciona al libro de Jevons por haber presentado una versión algebraica «mucho más compleja» de la oferta y la demanda a través de la utilidad ${ }^{20}$. Para Jenkin, en un mercado suficientemente grande las disposiciones a ofertar y demandar serán muy diferentes según cambien el precio y las expectativas ${ }^{21}$. Proyecta esta noción en un gráfico y presenta los excedentes del consumidor y del productor como las áreas situadas respectivamente entre la línea del precio de equilibrio y las curvas de la demanda y la oferta ${ }^{22}$.

\section{JENKIN Y LOS ECONOMISTAS}

Varios aspectos de la obra de Jenkin atrajeron la atención de Edgeworth: el método matemático y geométrico; la descripción de la oferta y la demanda

${ }^{18}$ Ibíd., pp. 92-3, 102.

19 Es su única obra económica disponible en castellano; Jenkin (1871-72), pp. 250-64.

20 Jenkin (1887), pp. 107-108.

21 Ibid., p. 109.

22 Declara que su análisis es novedoso, aunque en realidad Dupuit había expuesto la noción del excedente del consumidor en 1844. Jenkin detecta, como Cournot, que la clave de la incidencia tributaria reside en la elasticidad relativa. Jenkin (1887), pp. 113-115; Houghton (1958), p. 53; Humphrey (1992), p. 19. Hay un error en Jenkin (1887), p. 114, cuando el autor concluye que si el impuesto recae sobre los compradores la demanda es vertical: debe decir horizontal. 
como funciones, en línea con el análisis de Cournot; el énfasis en los procesos mentales (pero recuérdese que Jenkin no desarrolla la noción de utilidad); el excedente del consumidor; y en especial, como se destacó antes, la importancia de la capacidad de negociación en la determinación del precio, sobre todo en un mercado de competencia característicamente no perfecta, como el mercado de trabajo ${ }^{23}$.

No obstante, el pensamiento económico de Jenkin no iba a tener ni de lejos el reconocimiento que merecieron sus trabajos en otros campos. Una primera razón, apuntada por varios especialistas, es que Jenkin no era un economista profesional, aspecto que tiene una importancia creciente a finales del siglo XIX, cuando la economía se consolida como ciencia independiente. Así, es razonable que hubiese una fuerza interna que recelase en principio de los miembros de otra profesión, y sin duda Jenkin lo era: jamás pretendió ser primordialmente un economista. Al revés de Jevons, no decidió hacerse economista, y al revés de Marshall, no abrigó deseo alguno de fundar una nueva ortodoxia en una disciplina diferente de la suya.

La lista de autores cuyas contribuciones fueron tan importantes como ignoradas en su tiempo es extensa y conocida ${ }^{24}$. Pero Jenkin no fue simplemente ignorado. Lo que le distingue es el trato hostil que le dispensaron William Stanley Jevons y Alfred Marshall. Es posible conjeturar que esa escasa generosidad se debió a que Jenkin, al revés que Dupuit y Dionysius Lardner, por nombrar a dos ingenieros famosos por su obra económica, era contemporáneo de Jevons y Marshall y publicó antes que ellos un análisis con aspectos parecidos al del primero y llamativamente parecidos al del segundo. Pero conjeturas aparte, la hostilidad se puede probar y la escasa justificación de la misma también ${ }^{25}$.

Jevons conocía la obra de Jenkin y sentía por ella y por su autor un considerable respeto. En septiembre de 1874 le escribe a Walras, para agradecerle el ejemplar de los Elementos que le había enviado - «una obra altamente cien-

23 Edgeworth (1923) y Spengler (1971), p. 37.

${ }^{24}$ Hutchison (1967), p. 30, menciona a: Lloyd, Longfield, Cournot, Dupuit, John Rae, Auguste y Léon Walras, Von Thünen y otros. Entre los especialistas que han destacado el carácter de outsider de Fleeming Jenkin, puede mencionarse al autor del manual que más atención presta a Jenkin de entre todos los textos de historia del pensamiento económico que he podido consultar: Whittaker (1948), pp. 504-5.

25 Uno de mis evaluadores plantea el interrogante de por qué Dupuit resultó más admitido que Jenkin. Aparte de las razones y matizaciones alegadas en el texto, puede apuntarse aquí que Dupuit, al contrario que Jenkin, tuvo un trato frecuente con la profesión economica en su pais, a partir de su relación con el muy influyente Joseph Garnier. A pesar de que había reticencias hacia los ingenieros, Dupuit publicó en el Journal des Economistes y fue miembro de la Société d'Économie Politique y la Société d'Économie Sociale; cf. Etner (1987), pp. 116-121. 
tífica y original»-, aunque le aclara que no cree que atraiga el interés de más de media docena de expertos en todo el mundo: uno de los nombres que da es el de Fleeming Jenkin ${ }^{26}$.

Como es sabido, Herbert Stanley Jevons, hijo del economista, reveló que el artículo de Jenkin de 1870 fue el responsable de que Jevons se apresurara a concluir y publicar su Theory of political economy, lo que ocurrió en octubre del año siguiente: «Según una de las notas manuscritas de mi padre, la publicación pudo haber sido demorada hasta mucho después de 1871, de no haber sido por la aparición en 1868 y 1870 de los artículos del Profesor Fleeming Jenkin» 27.

Jevons no deseaba ser anticipado por Jenkin, con quien había mantenido relación epistolar a raíz del artículo sobre los sindicatos de 1868 , que Jenkin le remitió en forma manuscrita. Por desgracia, no se han conservado las cartas enviadas por Jevons, pero sí tres que le escribió Jenkin en marzo de ese año, que incluyen unos gráficos muy notables, de exactamente la misma factura que el célebre que Jevons pintó en el capítulo IV de su Theory para ilustrar el intercambio. Es posible que Jevons ya estuviera pensando en un gráfico de esas características - dos curvas de utilidad superpuestas - pero no hay forma de probarlo. Jenkin insiste en las dificultades de medición de la utilidad y en la indeterminación del precio en el monopolio bilateral ${ }^{28}$.

El recelo de Jevons ante la omisión de su nombre en el artículo de Jenkin sobre las leyes de la oferta y la demanda - donde no se nombra prácticamente a nadie aparte de Mill y Thornton- quizá pueda explicar el escaso reconocimiento de éste en la Theory. Su nombre no aparece cuando habla Jevons sobre W. T. Thornton ${ }^{29}$; lo menciona en el prólogo a la segunda edición como uno de los pocos «que se aventura en la ingrata cuestión de la ciencia matemáticoeconómica» ${ }^{30}$, y Jevons declara que su deuda mayor es con Lardner, a quien atribuye incorrectamente la ilustración gráfica de las leyes de la oferta y la demanda ${ }^{31}$.

Jevons no dibujó curvas de oferta y demanda, que obviamente había visto en el artículo de Jenkin de 1870, razón por la cual parecía razonable opinar, como hace Blaug con explícita referencia a Fleeming Jenkin: «Jevons estaba en deuda con un ejército de predecesores, pero no aprendió de ellos tanto como

26 Uemiya (1981), p. 175; Jevons (1977), vol. IV, p. 66.

27 Jevons (1977), vol. III, p. 166.

28 Ibid., pp. 167-178; Creedy (1992), pp. 119-20; Black (1962), pp. 199-200.

29 Jevons (1871), pp. 148-50.

30 Ibid., p. 65. Los otros nombres que da son el americano Simon Newcomb y los británicos Henry Dunning Macleod, George Darwin y Alfred Marshall.

31 Ibid., pp. 50-51; Uemiya (1981), pp. 181, 187. 
habria podidos ${ }^{32}$. Sin embargo, Bostaph y Shieh (1987) arguyen que esto es menos claro de lo que parece. Rastrean la historia hasta John Maynard Keynes, que menciona en su biografía de Jevons a un cuaderno de notas tomadas por un alumno en el que shay un bosquejo de una curva de demanda» ${ }^{33}$.

Dicho cuaderno ha sido publicado con el título de Lectures on Political Economy, 1875-1876, y alli puede verse a la curva en cuestión. Es explícitamente una curva de oferta con pendiente negativa, que representa el hecho de que cuando la oferta cae el precio aumenta. «Esta curva expresa la utilidad», dice Jevons. En otra lección, al hablar de la oferta y la demanda, parece tener presente a Jenkin cuando afirma que «son cantidades, no estados mentales». Y más adelante sostiene que no conocemos las funciones de utilidad, y tampoco las leyes de la oferta y la demanda, con lo que será necesario recurrir a reconstrucciones estadísticas ${ }^{34}$.

En la Theory subraya que «el precio es el único test de la utilidad» y que su teoría de la utilidad es «perfectamente coherente» con y «manifestación práctica» de la oferta y la demanda: «si pudiésemos determinar las funciones de utilidad, seria posible transformarlas para que expresaran claramente la equivalencia entre oferta y demandas ${ }^{35}$. Aun admitiendo todo esto, siguen siendo ciertas dos cosas que nadie ha podido justificar adecuadamente por razones de carácter técnico y no personal: primera, por qué Jevons no hizo algo aparentemente tan sencillo como dibujar dos curvas de oferta y demanda, y segunda, por qué no mencionó las diversas ideas de Jenkin que, como se ha visto en el apartado anterior, coincidian en alguna medida con las suyas. Jevons alegó -igual que Jenkin - que las curvas de oferta y demanda se podian derivar a partir de las funciones de utilidad. Admitió la validez del análisis de Jenkin pero insistió en que él ya empleaba curvas intersecantes para ilustrar el precio de mercado en sus lecciones antes de 1870 . No hay prueba alguna para esta reivindicación, como tampoco la habrá en el caso de Marshall ${ }^{36}$.

32 Blaug (1989), p. 308.

33 Keynes (1963), p. 294n.

34 Jevons (1977), vol, VI, pp. 16, 81, 88-9.

35 Jevons (1871), pp. 143, 148, 174.

36 Jevons (1977), vol. VI, p. x; Black (1987); Robertson (1985), p. 48; Staley (1989), p. 140; Brownlie y Lloyd Prichard (1963), pp. 215-6; Houghton (1958), p. 53n; Ekelund y Hébert (1992), pp. 329, 385; Uemiya (1981), pp. 184-85; Bostaph y Shieh (1987), pp. 122-24; White (1989), pp. 443-48. Uemiya (p. 186) conjetura que la razón última del trato poco cariñoso de Jevons hacia Jenkin se explica no porque éste se hubiese adelantado a aquél sino porque Jevons no queria verse mezclado con alguien que, al no basar su análisis en la utilidad, no confluía con él en el nuevo carril anti-clásico hacia donde deseaba impulsar a la teoria económica. En una línea similar argumenta White. Pero aunque parece razonable, esa reivindicación puede formularse perfectamente haciendo mención a Jenkin y sus gráficos. No se olvide que es el propio Jenkin el que 
La lectura de los Principios de Economía de Alfred Marshall, en su edición definitiva de 1920, da la pauta del reconocimiento que dispensaba el gran economista inglés a sus predecesores. Hay sólo una mención a Jenkin, en la nota final del capítulo 13 del libro 5. Marshall se limita a apuntar que su método es similar al de Dupuit y Jenkin, a quien cita mal y fecha en 1871 . Pese al notable parecido de sus análisis, no hay una sola mención a Jenkin cuando Marshall estudia la incidencia de los impuestos y el excedente del consumidor y del productor; ni tampoco en el Apéndice J sobre la doctrina del fondo de salarios. Los antecedentes de Marshall, tal cual queda consignado en el prólogo a la primera edición del libro, de 1890, son pocos: Cournot y Von Thünen -lejanos y, nótese, no británicos ${ }^{37}$.

La edición variorum de los Principios arroja alguna luz sobre este inadecuado reconocimiento de Jenkin por Marshall. Por lo pronto, en la primera edición, la nota mencionada en el párrafo anterior no incluye a Dupuit. Guillebaud, el editor, comenta que entre los papeles de Marshall hay una hoja, en la tercera edición del libro, de 1895, correspondiente a dicha nota, en la que Marshall dejó manuscritas estas líneas: «Las curvas de Fleeming Jenkin tienen una forma peculiar, y no se parecen a nada que yo conozca, a excepción de las que Rau añadió como apéndice a las primeras ediciones de su Volkswirtshatfslebre."

Marshall alega que conoció la obra de Karl Heinrich Rau antes de ver el trabajo de Jenkin (que cita bien), y aun antes había leído a Cournot y Von Thünen. La nota termina así:

La sustancia de las curvas de este capitulo ya estaba en mis lecciones de 1870, o antes: las curvas relativas al monopolio corresponden a una etapa muy posterior de mi trabajo, y fueron presentadas ante la Sociedad Filosófica de Cambridge en 1873. Él [Jenkin] no conoció mi obra, así como yo no supe del articulo que leyó en la Sociedad de Edimburgo [el de los impuestos] hasta muchos años después. Por fin él oyó hablar de mi trabajo y me envió un ejemplar de sus escritos ${ }^{38}$.

\footnotetext{
descarta explicitamente la relevancia empirica del enfoque subjetivo y la utilidad. Es interesante a este respecto que después Marshall se remitiese a Cournot, que se enrola aún más que Jenkin entre los no subjetivistas.

37 Marshall (1920), p. 394n. Marshall no cita el trabajo concreto de Jenkin. Sólo habla de «Edinburgh Philosophical Transactions» y 1871. Son dos errores. La fecha correcta es 1870 y aunque los articulos de 1868 y 1870 son de Edimburgo, ninguno corresponde a esa cita. «Marshall fue generoso de forma sistemáticamente exagerada con sus predecesores clásicos y más bien tacaño con sus contemporáneos»; Gordon, (1973), p. 21. «Marshall era muy consciente de su papel como lider nacional», Schumpeter (1954), p. 840.

38 Marshall (1961), vol. II, p. 534.
} 
Se observa, por tanto, que Marshall sabía muy bien quién era Jenkin, y en las ediciones anteriores de los Principios fue capaz de mencionarlo y hay testimonios de que estaba lo suficientemente familiarizado con sus escritos como para citarlos correctamente. No obstante, en ningún caso reconoce anticipación alguna. Además, esta nota no menciona al excedente del consumidor.

El primer trabajo publicado por Marshall fue una recensión de la Theory de Jevons, en abril de 1872. Marshall tenía treinta años de edad. En esa reseña, aparte de Jevons, Marshall cita a un solo autor contemporáneo: Fleeming Jenkin. Característicamente, disputa Marshall la utilidad del método matemático. Se inclina por utilizar palabras que todos puedan entender y prefiere «el lenguaje de los diagramas o, como ha sido llamado por el Profesor Fleeming Jenkin, la representación gráfica» ${ }^{39}$. La importancia que Marshall asigna a Jenkin era, entonces, indisputable. Y recuérdese el consejo que le dio aquél a Jevons: el libro habria mejorado sin las matemáticas y con los diagramas.

Con el paso del tiempo la generosidad de Marshall disminuyó. En 1908 le confiesa a John Bates Clark que se ha vuelto «insensible»: dice que se limita a hacer referencias en notas al pie de página, lo que en el caso de Jenkin es falso, y no admite deudas, lo que en cualquier caso es cómodo:

Mi regla ha sido citar al pie de página a cualquiera que según mi conocimiento haya dicho una cosa antes de que yo la haya publicado, aunque yo la haya presentado en mis lecciones muchos años antes de saber que se le habia ocurrido a él. Sólo cito, no hago mención alguna a deudas en ningün sentido; soy consciente de que algunos pensarán que implicitamente reconozco deudas. Ejemplos de éstas son Francis Walker y Fleeming Jenkin ${ }^{40}$.

En los años cruciales, en torno a 1870, Alfred Marshall no publica nada. Hubo sin embargo varios manuscritos, borradores de eventuales publicaciones, que leyeron sus alumnos y colegas más aventajados: Herbert Somerton Foxwell (el después sir), Henry Hardinge Cunynghame y John Neville Keynes. Ellos apoyaron las reivindicaciones del maestro. En la biografía que escribió sobre él, John Maynard Keynes descarta toda posible anticipación de nadie a Marshall ${ }^{41}$.

39 Pigou (1925), p. 99.

40 Ibíd., p. 416. Las muestras de la escasa generosidad de Marshall son abundantes. Pueden mencionarse los ejemplos de Hans von Mangoldt (1824-1868), Dupuit y Léon Walras (18341910); cf. Schneider (1960); Segura (1987 y 1988); Schumpeter (1954), pp. 838-40 y 1061; y Houghton (1958), p. 53n. Y no hay que olvidar a Jevons, sobre el que Keynes ha escrito: «Marshall era extraordinariamente reacio a admitir que le debia algo a Jevons», Keynes (1963), p. 287; Jevons (1871), p. 34, y Bostaph y Shieh (1987), pp. 120-21.

41 Para Keynes «la idea de aplicar métodos matemáticos [a la economía] estaba en el aire, 
Marshall insiste en que la base de su teoría ya estaba terminada antes de la Theory de Jevons, o sea, antes de 1871, y que había trabajado sobre la productividad marginal en 1869 y sobre la cuasi-renta en 1868. Le escribió a Léon Walras en 1883 , diciéndole que había enseñado la teoria de la utilidad marginal en Cambridge antes de la Theory y que, siguiendo a Cournot, había ido más allá que Jevons. En lo referido al excedente del consumidor, le escribe a E. R. A. Seligman en 1896: «mis deudas son con Cournot, no con Fleeming Jenkin ni con Dupuit» ${ }^{42}$.

La actitud de Marshall es importante para explicar el eco de la obra de Jenkin, puesto que el gran economista de Cambridge, al revés que Jevons, aspiraba a fundar y fundó una escuela, un nuevo clasicismo en economía. Es revelador que ya en 1879 los discípulos de Marshall procurasen blindar al maestro ante las eventuales críticas y reivindicaciones que podrían plantearse por «fundar» una escuela veinte años después de la aparición de los textos básicos de la misma, firmados por otras personas.

En noviembre de ese año, se entabla un cuidadoso intercambio epistolar entre Foxwell y Jevons. En un momento dado, Foxwell deja caer que el trabajo de Marshall fue «contemporáneo» al de Jevons y menciona a las lecciones de Marshall en 1869-1876. Jevons le responde que no discute prioridades, pero acto seguido se cuida bien en aclarar que él había expuesto públicamente su teoría en 1862, es decir, mucho antes de que Marshall entrara en escena. Zanjada esta cuestión, Jevons despide el problema arguyendo que no tiene sentido disputar prioridades «una vez conocidos los textos de autores anteriores a todos, como Gossen, Dupuit y Cournot» ${ }^{43}$.

En el caso de Jenkin, el responsable de la edición de los escritos económicos tempranos de Marshall, Whittaker, cree que la influencia de Jenkin sobre Marshall fue imperceptible. Quizá haya sido así, pero no está claro que Jenkin no se le haya anticipado. Este es el punto en cuestión: no que Marshall haya copiado a Jenkin sino que arribó a un análisis similar después.

Es perfectamente posible que, como reconoce en un momento el propio

pero todavia [hasta Marshall] no había producido nada sustancial» y «en 1867 [Marshall] empezó a desarrollar los métodos diagramáticos»; Keynes (1963), pp. 150 y 153. Schumpeter (1954) comenta: «Una y otra vez me ha impresionado el hecho de que economistas competentes e incluso eminentes incurran en la acrítica costumbre de atribuir a Marshall lo que, «objetivamente», debería ser atribuido a otros (jincluso la curva de la demanda «marshallianaw!)», p. 839n.

42 Marshall (1975), vol. 1, p. 39. Ekelund y Gramm (1970) argumentan que la importancia de Dupuit en la formulación de la demanda marshalliana es equivalente a la de Cournot, y fue en consecuencia mayor de lo que el propio Marshall admite.

${ }^{43}$ Jevons (1977), vol. V, pp. 78, 80. Cf. también Ekelund y Shieh (1989), p. 26n. 
Marshall, obtuviesen sus conclusiones de forma independiente ${ }^{44}$. Después de todo, ambos tenian formación matemática y estaban aplicándola al texto básico de economía de entonces, los Principios de Stuart Mill. La formulación simétrica de oferta y demanda es un descubrimiento lógico, como apunta Whitaker, para quien estuviese desarrollando el pensamiento de Mill. El propio Mill se acerca a la solución en su reseña de Thornton de 1869, pero Jenkin, como se vio antes, había planteado la solución formalmente correcta en $1868^{45}$. Este año es una fecha importante para esta cuestión, porque es el año en que Marshall declaró haber leído a Cournot. Y hay que recordar que, al parecer, Jenkin no había leído más que a Stuart Mill hasta 1870 . No conocía a Cournot y no tenía en principio por qué estar al tanto de los artículos de $\mathrm{Du}$ puit, puesto que sus intereses en el campo de la ingeniería iban por otros derroteros que los que habian preocupado a los ingenieros-economistas de la Escuela de Puentes y Caminos de Paris en la década de 1840 -más extraño, quizá, es que no citara a D. Lardner.

Las lecciones de Marshall nos brindan otro testimonio interesante, en una carta que Foxwell escribe a Maynard Keynes en abril de 1925:

Lo lei [al artículo de Jenkin] en las vacaciones de Semana Santa de 1870, en la época en que asistia a las clases de Marshall sobre economia diagramática, y se lo mostré. Nunca olvidaré su expresión de disgusto mientras hojeaba el artículo. Es que no había nada en Cournot que se aproximase tanto al enfoque general de Marshall sobre la teoria del valor, y en particular a su presentación de la ecuación de oferta y demanda ${ }^{46}$.

Hay razones para conjeturar, entonces, que la importancia de Jenkin para Marshall fue mayor que la reflejada en los Principios. Pero falta la prueba de que Marshall se le había anticipado independientemente. Su propio testimonio y el de sus alumnos asi lo sugiere. A Marshall le disgusta ver el articulo de Jenkin e insiste en que no le aportó nada que no supiera - en ambos puntos coincide con Jevons-. Ello no es imposible, pero su anticipación a Jenkin no se puede apoyar en nada que no sea meramente testimonial 47.

44 Marshall (1961), vol. I, p. 476; Creedy (1992), p. 68.

45 Bradley (1989), p. 53.

46 Marshall (1975), p. 45n.

47 Ibid., vol. 2, p. 241. La simpatía de Whittaker por Marshall se observa en este breve comentario: «Parece probable que Jenkin haya sido anticipado por Leslie Stephen, que era como Marshall un Wrangler de Cambridge», p. 41 . Esta conjetura, basada en un inédito cuaderno de notas de Stephen, que Whittaker cita a través del biografo de aquél, es poco defendible. Sir Leslie Stephen, distinguido intelectual, padre de Vanessa Bell y Virginia Woolf, no fue un economista ni un científico, sino un hombre de letras - su biógrafo, Frederic William Maitland, fue un histo- 


\section{CONCLUSIONES}

Fleeming Jenkin fue un economista notable, que con su bagaje de conocimientos de ingeniería fue capaz de un interesante trabajo científico en economia. Entre sus avances destacan el análisis algebraico y gráfico de la oferta y la demanda como funciones, los mercados imperfectamente competitivos, y el excedente del consumidor y del productor.

Vistos sus logros, la indiferencia con que fue tratado por sus contemporáneos - con la excepción de Edgeworth- carece de justificación. Es verdad que Jenkin fue un outsider de la economía, que empezaba entonces a institucionalizarse como profesión. Pero no se puede pasar por alto la actitud que tuvieron hacia él Jevons y Marshall. Ambos devaluaron marcadamente sus aportaciones e insistieron en que habian trabajado independientemente de Jenkin y en que le habian precedido en todo lo que importaba. Adoptaron esta última actitud con tanto entusiasmo como pocas pruebas.

\section{BIBLIOGRAFİA}

BlaCK, R. D. Collison (1962): «W. S. Jevons and the economists of his time», Manchester School, vol. 30, núm. 3, pp. 203-21; reimpreso en John Cunningham Wood (ed.), William Stanley Jevons. Critical Assessments, Londres y Nueva York, Routledge, vol. I. - (1987): «Jenkin, Henry Charles Fleeming», The New Palgrave.

Blaug, Mark (1989), Economic theory in retrospect, 4." ed., Cambridge University Press

[trad. cast. de la 3.a ed. FCE].

BostapH, Samuel, y SHIEH, Yeung-Nan (1987): «Jevons's demand curve», History of Political Economy, vol. 19, núm. 1, pp. 107-26.

Bradley, Michael E. (1989): «John Stuart Mill's demand curves», History of Political Economy, vol. 21, núm. 1, pp. 43-56.

Brownlie, A. D., y Lloyd Prichard, M. F. (1963): «Professor Fleeming Jenkin, 1933.

1885. Pioneer in engineering and political economym, Oxford Economic Papers, vol.

15, pp. 204-16.

CREEDY, John (1992): Demand and exchange in economic analysis, Aldershot, Edward Elgar.

riador. En una carta Stephen revela poco aprecio por Thornton, «que siempre está aburriendo a todo el mundo con la oferta y la demanda». Maitland (1906), pp. 45-6, 73, 75, 189. Entre los colegas de Stephen en Cambridge se contaba William Whewell, un notable pionero de la economía matemática -incidentalmente, Marshall no lo menciona jamás-. Los interesantes trabajos de Whewell, un matemático tan cauteloso como Marshall sobre las posibilidades de las matemáticas en la economia, pueden verse en Whewell (1829). Hutchison sostiene que los analisis de Whewell «dificilmente pueden haber sido desconocidos por Marshall»; Hutchison (1967), p. 77, y la extensa nota 4, pp. 77.79. 
EdGeworth, Francis Ysidro (1923): «Jenkin, Henry Charles Fleeming», Palgrave's Dictionary of Political Economy, Londres.

Ekelund, Robert B. Jr., y HeBert, R. F. (1992): Historia de la teoría económica y de su método, Madrid, McGraw-Hill.

Ekelund, Robert B. Jr., y Gramm, William P. (1970): «Early French contributions to Marshallian demand theory», Southern Economic Journal, vol. 36, núm. 3, pp. 277-86.

ExElund, Robert B. Jr., y SHIEH, Yeung-Nan (1989): «Jevons on utility, exchange, and demand theory: a reassessment», Manchester School, vol. 57, núm. 1, pp. 17-33.

Ekelund, Robert B. Jr., y ThOMmesen, Sven (1989): «Disequilibrium theory and Thornton's assault on the laws of supply and demand», History of Political Economy, vol. 21, núm. 4, pp. 567-92.

ETNER, François (1987): Histoire du calcul économique en France, Paris, Economica.

ForGET, Evelyn L. (1991): «J. S. Mill, F. D. Longe and W. T. Thornton on demand and supply», Joumal of the History of Economic Thought, vol. 13, núm. 2, pp. 205-21.

GoRDON, Scott (1973): «The wage-fund controversy: the second round», History of Political Economy, vol. 5, nüm. 1, pp. 14-35.

Groenewegen, Peter D. (1994): «Alfred Marshall and the Labour Commission 18911894", European Journal of the History of Economic Thought, vol. 1, núm. 2, pp. 273-96.

Houghton, R. W. (1958): «A note on the early history of consumer's surplus», Economica, N.S., vol. 23, pp. 49-57.

HOWEY, R. S. (1989): The rise of the marginal utility school, 1870-1889, Columbia University Press.

HumphreY, Thomas M. (1992): «Marshallian cross diagrams and their uses before Alfred Marshall: the origins of supply and demand geometry», Federal Reserve Bank of Richmond Economic Review, vol. 78, núm. 2, pp. 3-23.

HuTCHISON, T. W. (1967): Historia del pensamiento económico, 1870-1929, Madrid, Gredos.

HuTT, W. H. (1936): Economists and the public, Londres, Jonathan Cape.

JENKIN, Fleeming (1871-72, 1964): «Sobre los principios que regulan la incidencia de los impuestos», Richard A. Musgrave y Carl S. Shoup (eds.), Ensayos sobre economía impositiva, México, Fondo de Cultura Económica.

-_ (1887, 1931): Papers, Literary, Scientific, \&c., S. Colvin y J. A. Ewing (eds.), Londres, vol. II; reimpreso como: The graphic representation of the laws of supply and demand, and other essays on political economy, Londres, The London School of Economics and Political Science, Series of Reprints of Scarce Tracts in Economic and Political Science, nüm. 9.

Jevons, William Stanley (1871, 1970): Theory of political economy, R. D. C. Black (ed.), Harmondsworth, Penguin.

- (1977): Papers and Correspondence of William Stanley Jevons, R. D. Collison Black (ed.), Londres, Macmillan.

KEYNES, John Maynard (1963): Essays in Biography, Nueva York, W. W. Norton [trad. cast. Crítica].

Martland, Frederic William (1906): The Life and Letters of Leslie Stephen, Londres, Duckworth.

Marshall, Alfred (1920, 1977): Principles of Economics, 8." ed., Londres, Macmillan [trad. cast. Aguilar].

(1961): Principles of economics, 9." ed. variorum, C. W. Guillebaud (ed.), Londres, Macmillan. 
(1975): The early economic writings of Alfred Marsball, 1867-1890, J. K. Whitaker (ed.), Londres, Macmillan.

Mill., John Stuart (1967): Essays on Economics and Society, J. M. Robson (ed.), Toronto y Londres, University of Toronto Press y Kegan Paul.

Negishi, Takashi (1986): «Thornton's criticism of equilibrium theory and Mill», History of Political Economy, vol. 18, núm. 4, pp. 567-77.

O'BRIEN, D. P. (1989): Los economistas clásicos, Madrid, Alianza.

Petridis, Anastasios (1973): «Alfred Marshall's attitudes to and economic analysis of trade unions: a case of anomalies in a competitive system», History of Political Economy, vol. 5, núm. 1, pp. 165-98.

PIgou, Arthur Cecil (ed.) (1925, 1966): Memorials of Alfred Marshall, Nueva York, Kelley.

Robertson, R. M. (1985): “Jevons y sus precursores», Cuadernos Económicos de ICE, núm. 29 , pp. 37-54.

SCHNeIder, Erich (1960): «Hans von Mangoldt on price theory: a contribution to the history of mathematical economicsm, Econometrica, vol. 28, núm. 2, pp. 380-92.

SChumpeter, J. A. (1954): History of economic analysis, Nueva York, Oxford University Press [trad. esp. Ariel].

SCHWARTZ, Pedro (1972): The new political economy of J. S. Mill, Londres, Weidenfeld and Nicolson [trad. esp. Tecnos].

Segura, Julio (1987): «La obra de Walras al cabo de un siglo», introducción a los Elementos de L. Walras, Madrid, Alianza.

- (1988): «Léon Walras y la historiografía del pensamiento económico», Revista de Historia Económica, año VI, núm. 1, pp. 73-102.

SMITH, Adam (1776, 1994): La riqueza de las naciones, Madrid, Alianza.

SPENGLER, J. J. (1971): «El problema del orden en los asuntos económicos», J. J. Spengler y W. R. Allen (eds.), El pensamiento económico de Aristóteles a Marshall, Madrid, Tecnos.

STALEY, C. E. (1989): A bistory of economic thought, Oxford, Basil Blackwell.

StEvenson, Robert Louis $(1887,1924)$ : «Memoir of Fleeming Jenkin», The Works of Robert Louis Stevenson, Londres, Tusitala, vol. XIX.

UemiYA, S. (1981, 1988): «Jevons and Fleeming Jenkin», Kobe University Economic Review, vol. 27, pp. 45-57; reimpreso en John Cunningham Wood (ed.), William Stanley Jevons. Critical Assessments, Londres y Nueva York, Routledge, vol. III.

WHEWELL, William $(1829,1831$ \& 1850; 1971): Mathematical exposition of some doctrines of political economy, Nueva York, Kelley.

WHITE, Michael V. (1989): «Why are there no supply and demand curves in Jevons?», History of Political Economy, vol. 21, núm. 3, pp. 425-56.

WhITTAKER, Edmund (1948): Historia del pensamiento económico, México, Fondo de Cultura Económica. 